\title{
CCD PHOTOMETRY OF NGC 2453
}

\author{
D.C.V. MALLIK, RAM SAGER and A.K. PATI \\ Indian Institute of Astrophysics, Bangalore 560034, India
}

We have undertaken a detailed photometric study of the open cluster NGC 2453 in Puppis with a view to determining accurately its distance modulus and to investigate its possible association with the PN NGC 2452. We have observed the core region of the cluster in V and I photometric bands using a Thomson-CSF TH 7882 CCD chip with the format $384 \times 576$ pixels, at the $\mathrm{f} / 13$ cassegrain focus of the 102-cm telescope at Vainu Bappu Observatory, Kavalur, India. Each image frame covers a field of $2^{\prime} .3 \times 3^{\prime} .4$ of the sky. Bias frames and flat field exposures on the twilight sky were obtained as per standard practice.

The data were initially processed using STARLINK package and the magnitude estimation of a star has been done using DAOPHOT. The figure below shows a V, V-I plot of the core region of the cluster. A total of 275 stars have been plotted in the diagram. We find a well populated main sequence down to $V \geq$ $20^{m} .0$. This is the first time the unevolved main sequence of the cluster has been observed. We have adopted a $E(B-V)=0^{m} .47$ from earlier work and used $A_{V}=3.06 * E(B-V)$ to correct for interstellar extinction. ZAMS from Walker (1985, MNRAS 213, 889) was used to fit the cluster CMD after applying a color correction $E(V-I)=1.25 E(B-V)$. A true distance modulus of $12^{m} .9 \pm 0^{m} .25$ has been obtained which puts the cluster at a distance of $3.80 \pm 0.47 \mathrm{kpc}$. The PN NGC 2452 has a directly determined extinction distance of $3.57 \pm 0.47 k p c$ (Gathier 1984, Ph.D. thesis, Groningen).

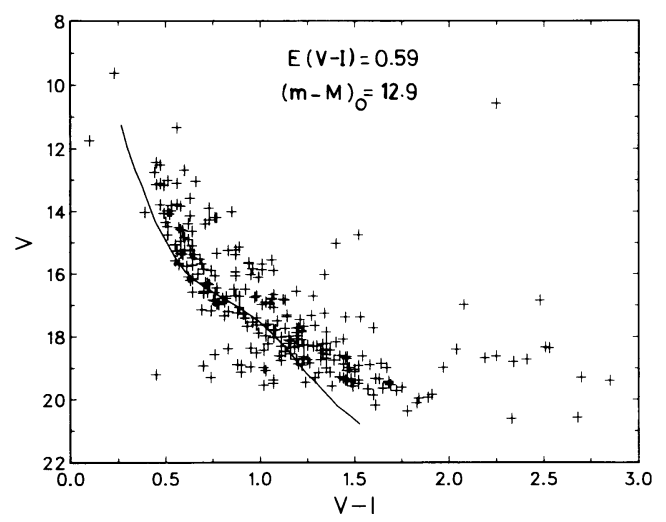

